\title{
Alternative end-joining mechanisms: a historical perspective
}

\section{Anabelle Decottignies*}

Genetic and Epigenetic Alterations of Genomes, de Duve Institute, Faculty of Pharmacy and Biomedical Sciences, Catholic University of Louvain, Brussels, Belgium

\section{Edited by:}

Antonio Porro, École Polytechnique Fédérale de Lausanne, Switzerland

\section{Reviewed by:}

Bjoern Schwer, Boston Children's Hospital, Harvard Medical School, USA

Sascha Feuerhahn, École

Polytechnique Fédérale de Lausanne, Switzerland

\section{${ }^{*}$ Correspondence:}

Anabelle Decottignies, Genetic and Epigenetic Alterations of Genomes, de Duve Institute, Faculty of Pharmacy and Biomedical Sciences, Catholic University of Louvain,

Avenue Hippocrate $75+1,1200$ Brussels, Belgium.

e-mail: anabelle.decottignies@ uclouvain.be
In the presence of functional DNA repair pathways, DNA double-strand breaks (DSBs) are mainly repaired by non-homologous end-joining (NHEJ) or homologous recombination (HR), two conserved pathways that protect cells from aberrant chromosomal rearrangements. During the past two decades however, unusual and presumably distinct DNA end-joining repair activities have been unraveled in NHEJ-deficient cells and these are likely to operate in various chromosomal contexts and species. Most alternative DNA end-joining events reported so far appear to involve microhomologous sequences and are likely to rely on a subset of HR enzymes, namely those responsible for the single-strand annealing mechanism of HR, and on DNA Ligase III. Usually, microhomologies are not initially present at DSB ends and thus need to be unmasked through DNA end resection, a process that can lead to extensive nucleotide loss and is therefore highly mutagenic. In addition to microhomology-mediated end-joining events, recent studies in mammalian cells point toward the existence of a distinct and still ill defined alternative end-joining pathway that does not appear to rely on pre-existing microhomologies and may possibly involve DNA Ligase I. Whether dependent on microhomologies or not, alternative DNA end-joining mechanisms are likely to be highly mutagenic in vivo, being able to drive telomere fusion events and cancer-associated chromosomal translocations in mouse models. In the future, it will be important to better characterize the genetic requirements of these mutagenic alternative mechanisms of DNA end-joining.

Keywords: double-strand break, non-homologous end-joining, alternative end-joining, microhomology-mediated end-joining, single-strand annealing, chromosomal translocation, telomere fusion, Ku70/Ku80

\section{INTRODUCTION}

Double-strand breaks (DSBs) represent major threats to genome integrity. They can be induced during normal metabolism or may result from the presence of exogenous genotoxic agents like ionizing radiations or chemotherapeutic drugs. Cells have evolved two main pathways to repair these lesions: the non-homologous end-joining (NHEJ) pathway, that ensures direct resealing of DNA ends; and the homologous recombination (HR) pathway that relies on the presence of homologous DNA sequences for DSB repair. Repair through HR is not defined by a unique mechanism but operates through various mechanistically distinct DSB repair processes including synthesis-dependent strand annealing (SDSA), double Holliday junction resolution, and single-strand annealing (SSA; Paques and Haber, 1999; Chapman et al., 2012; Figure 1). The common step for HR-dependent DSB repair mechanisms is the initial formation of single-stranded DNA (ssDNA) for pairing with homologous DNA template sequences. These HR-dependent mechanisms of DSB repair have been extensively reviewed previously and will not be detailed here (Paques and Haber, 1999; Chapman et al., 2012).

Until very recently, how cells choose between NHEJ and HRdependent pathways for DSB repair was still unclear, although both the cell cycle stage and the nature of DSB ends were previously involved (Symington and Gautier, 2011; Chapman et al.,
2012). A critical determinant for the choice is provided by the $5^{\prime}-3^{\prime}$ resection of DNA ends that, while triggering HR-dependent repair, prevents NHEJ. On the contrary, direct binding at DSB ends of the conserved $\mathrm{Ku} 70 / \mathrm{Ku} 80$ heterodimer, a key complex of the NHEJ pathway that protects DNA ends against exonucleases, represses HR-dependent mechanisms (Pierce et al., 2001). Four studies recently shed new light on this important question of initial choice between NHEJ and HR for DSB repair and provided consistent evidences in favor of the existence of a tightly regulated mechanism (Chapman et al., 2013; Di Virgilio et al., 2013; Escribano-Diaz et al., 2013; Zimmermann et al., 2013). Four proteins were shown to play critical roles in repair pathway choice: RIF1 (Rap1-interacting factor 1), 53BP1 (p53 binding protein 1), BRCA1 (breast cancer type 1 susceptibility protein), and CtIP ( $\mathrm{C}$ terminus-binding protein-interacting protein). Briefly, it appears that, while 53BP1-RIF1 stimulates NHEJ, BRCA1 and CtIP together promote DNA end resection and HR. RIF1 is indeed recruited to DSB ends via an interaction with 53BP1 and both proteins cooperate to promote NHEJ in G1 cells. During G2, RIF1/53BP1 binding to DNA ends is repressed by BRCA1, ensuring a switch to HR during this stage of the cell cycle. Collectively, these studies provide strong evidences in favor of well-regulated competitions between NHEJ and HR pathways at DNA ends. 


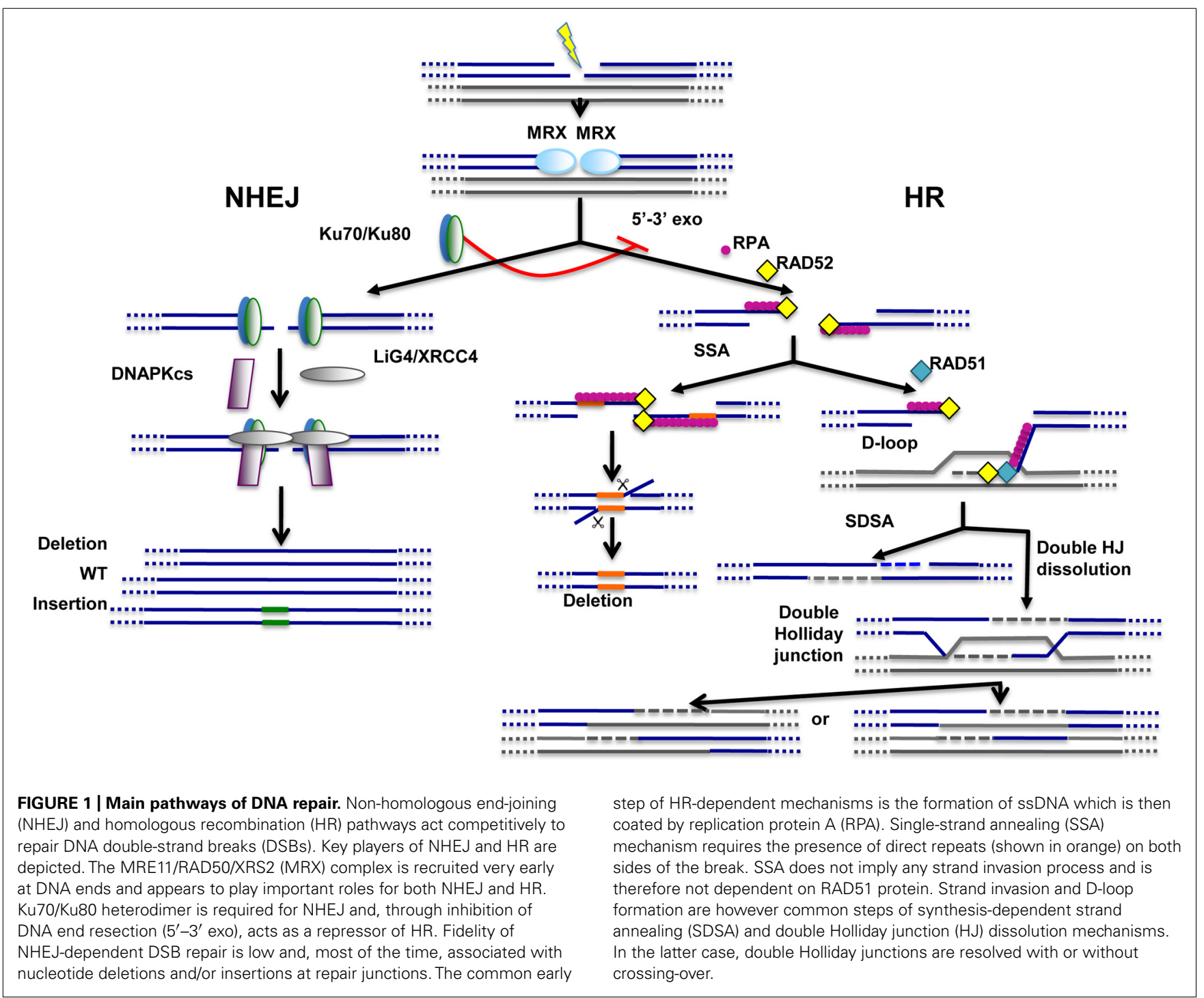

The choice of DSB repair pathway inevitably impacts on the fidelity of repair. Indeed, while HR is generally viewed as a conservative DSB repair pathway, NHEJ operates with poor fidelity and nucleotide deletions and/or insertions are frequently detected at repair junctions (Figure 1). However, not all HR-dependent mechanisms display high fidelity of repair. Namely, the SSA mechanism requires annealing at two directly repeated DNA sequences near DSB ends. Consequently, intervening nucleotides initially present between the direct repeat sequences flanking the break are lost during SSA-mediated repair (Figure 1).

More recently, backup pathways for DNA repair were identified in NHEJ-deficient cells of various organisms. Although it is still unclear whether only one or several backup repair pathways exist, they do not rely on large homologous DNA templates such as those involved in HR-dependent repair events and are therefore referred to as "alternative end-joining" pathways. However, alternative end-joining mechanisms usually -but not alwaysrely on the presence of microhomologies at or near DSB ends, suggesting that these repair events may not be entirely distinct from HR-dependent mechanisms. Here, I review the history of alternative end-joining discovery and the recent evidences that these alternative end-joining events are able to drive class switch recombination in the immune system, telomere fusions and chromosomal translocations in vivo.

\section{ALTERNATIVE END-JOINING REPAIR: ONE OR MORE BACKUP PATHWAY(S)?}

Proteins required for NHEJ include -but are not restricted tothe highly conserved Ku70/Ku80 heterodimeric complex, DNAdependent protein kinase catalytic subunit (DNA-PKcs) and DNA Ligase IV (LIG4) in complex with XRCC4 (Weterings and Chen, 2008). By directly binding DNA ends, Ku70/Ku80 ensures protection against exonucleases and, as such, acts as an inhibitor of HR (Figure 1). In 1996, thanks to the use of Ku70-deficient budding yeast mutants, Boulton and Jackson provided the first evidences for the existence of an alternative DNA end-joining pathway. This pathway was about 20 -fold less efficient than 
NHEJ and repair junctions displayed both nucleotide deletions and overlapping microhomologies of 3-16 nucleotides (Boulton and Jackson, 1996). Although it was known at that time that short microhomologous regions of up to five nucleotides were commonly recovered at NHEJ repair junctions of mammalian cells (Roth etal., 1985), this DNA repair pathway was clearly able to operate in a NHEJ-deficient background. Supporting the existence of a new DNA end-joining pathway, biochemical fractionation of calf thymus extracts yielded two fractions with distinct DSB repair activities (Mason et al., 1996). One fraction, presumably enriched for microhomology-mediated end-joining (MMEJ) activity, was clearly relying on the presence, on both sides of the DSB, of short repeat sequences. The second fraction, containing the NHEJ activity, was characterized by the presence of a DNA fill-in activity -inhibited by DNA polymerase inhibitorsand the ability to perform ligation of non-homologous DNA fragments (Mason etal., 1996). In agreement with the previous suggestion that very short sequence homologies are likely to help DNA end alignment prior to NHEJ-dependent repair (Roth etal., 1985; Roth and Wilson, 1986), some repair junctions produced by the NHEJ activity-containing fraction were also characterized by the presence of overlapping microhomologies. However, the remaining repair junctions were devoid of any microhomology, indicating that microhomologies were not strictly required for NHEJ in this system (Mason et al., 1996). Later on, backup pathways of end-joining were identified in various NHEJ-deficient mammalian cells (Kabotyanski et al., 1998; Feldmann et al., 2000; Wang et al., 2003).

Whether this newly identified backup MMEJ pathway was involving a new set of DNA repair proteins was unclear at that time. In 1994, and although they were working in a NHEJ-proficient budding yeast background, the group of Haber first postulated that microhomology-mediated DNA repair events may occur through a RAD52-dependent SSA-type mechanism (Kramer et al., 1994). The same group then reported that the lower limit for SSAdependent DSB repair was lying between 5 and 29 bp of homology, showing that sequence homologies may be very low for $\mathrm{HR}$, at least in budding yeast (Sugawara et al., 2000). They suggested however, that some differences may exist between classical SSA, involving large direct repeats, and "micro-SSA", in which homology lengths are much lower, as the latter process appeared to rely mostly on RAD59, a budding yeast homolog of RAD52, instead of RAD52 itself (Sugawara et al., 2000). Altogether, studies by the group of Haber thus pointed toward the possible contribution of HRdependent pathways in budding yeast MMEJ, suggesting that this may not represent a new completely distinct DNA repair pathway but could reflect a micro-SSA-type mechanism of DSB repair. In complete agreement with these predictions, a study performed in $X$. laevis eggs established that a purified fraction displaying MMEJ activity contained DNA Ligase III (LIG3), DNA polymerase $\varepsilon$, FEN-1 endonuclease, and exonuclease activities of $5^{\prime}-3^{\prime}$ and $3^{\prime}-5^{\prime}$ directionality and that the same fraction was able to process SSA intermediates (Gottlich et al., 1998). Next, it was reported that, in a NHEJ-deficient $S$. cerevisiae background, MMEJ events were not dependent on RAD52 but required the MRE11/RAD50/XRS2 complex previously implicated in both NHEJ and HR (Ma et al., 2003; Yu and Gabriel, 2003). The requirement for RAD59 was however, not tested. Extrachromosomal DSB repair experiments in NHEJ-deficient fission yeast mutants then provided additional evidences in favor of a SSA-dependent mechanism for MMEJ (Decottignies, 2007). In this system, both RAD22, the fission yeast RAD52 homolog, and EXOl, the $5^{\prime}-3^{\prime}$ exonuclease involved in the formation of ssDNA intermediates for HR, were required for MMEJ (Decottignies, 2007). However, another study conducted in mouse ES cells concluded that, although the first steps may be shared, alternative NHEJ in ES cells may be distinct from SSA during the late steps of repair (Bennardo et al., 2008). This conclusion came from the observation that mouse RAD52 was not able to stimulate alternative NHEJ in their experimental chromosomal context although the protein was able to promote SSA when the entire coding sequence of GFP was involved in homologydirected repair (Bennardo et al., 2008). One possibility however, would be that the annealing process may require another protein than RAD52, similarly, to the situation in budding yeast where RAD59, a RAD52 homolog, is required for annealing when only very short homologous sequences are available for SSA (Sugawara et al., 2000). In support of this, observations in RAD52 knock-out mouse models suggested that mouse RAD52 may only be involved in certain types of DSB repair processes while other HR-dependent events may be catalyzed by distinct proteins functionally related to RAD52 (Rijkers et al., 1998). This remains to be tested experimentally.

Additional proteins, whether from yeast or from higher eukaryotes, were reported to play a role in MMEJ. POL4, a member of the PolX family of polymerases with gap-filling activity, and proteins from the mismatch repair pathways were found to be required for MMEJ-dependent repair of substrates with non-perfect microhomologies in fission yeast (Decottignies, 2007). The MRE11 complex was found to be required for MMEJ in budding yeast (Ma et al., 2003), Arabidopsis (Heacock et al., 2004) and human cells (Delia-Maria et al., 2011), but dispensable for fission yeast MMEJ using an extrachromosomal DSB repair assay (Decottignies, 2007). It is believed however, that fission yeast MRE11 complex may be required for MMEJ events in a chromosomal context and/or for intermolecular MMEJ-dependent ligations (Decottignies, 2005, 2007). As stated above, first evidences for the involvement of LIG3 in the MMEJ process were provided by biochemical fractionation of X. laevis egg extracts (Gottlich etal., 1998). LIG3 contribution to MMEJ was later confirmed in HeLa cells (Wang et al., 2005), in human HTD114 cell line (Liang et al., 2008) and in mice (Simsek et al., 2011).

In mature mouse $\mathrm{B}$ cells activated by antigens, recent in vivo evidences indeed support the existence of a powerful backup mechanism able to compensate for NHEJ during immunoglobulin class switch recombination (CSR; Soulas-Sprauel et al., 2007; Yan et al., 2007). Whether this in vivo backup mechanism is similar to the MMEJ repair pathway described above is however still a matter of debate. In favor of this hypothesis, the backup CSR activity detected in the absence of either XRCC4 or LIG4 was found to operate through the recognition of microhomologies at DNA break borders and, in agreement with two previous reports (Audebert et al., 2004; Wang et al., 2005), was proposed to rely on XRCC1/LIG3 complex (Yan et al., 2007). Interestingly, XRCC1 was previously involved in SSA (Stark et al., 2004), further 
supporting the view that alternative NHEJ may similarly operate through a micro-SSA-like mechanism in immune cells. A more recent study published by the group of Jasin reported that, similarly to what happens in human cells in culture, mouse LIG3 is involved in an alternative end-joining pathway operating through annealing at pre-existing microhomologies (Simsek et al., 2011). They proposed that LIG4 was acting as a repressor of the DNA end resection activity required to produce the complementary ssDNA ends.

Although the studies reported above in various eukaryotic species converged onto the identification of the alternative endjoining backup pathway as a microhomology-dependent mechanism presumably relying on LIG3, recent data led to revise this view. Indeed, experiments performed in mammalian cells suggested the existence of an additional alternative end-joining pathway presumably relying on Ligase I (LIG1) and able to repair DSBs independently of pre-existing microhomologies (Boboila et al., 2010a,b, 2012; Simsek etal., 2011). First in vivo evidences came from the observation that, in either $K U 70^{-/-}$or $K U 70^{-/-} / L_{G} 4^{-/-}$mice, CSR appears to operate through two distinct alternative end-joining mechanisms in B cells, with only one relying on microhomologies (Boboila et al., 2010a). This newly unraveled alternative end-joining mechanism was not initially detected in either $L I G 4^{-/-}$or XRCC4 ${ }^{-/-}$mouse B cells for which microhomologies were recovered at all CSR junctions, suggesting that it may be repressed by $\mathrm{Ku} 70 / \mathrm{Ku} 80$. Further support in favor of the existence of a second alternative end-joining mechanism not relying on pre-existing microhomologies was provided by sequencing of chromosomal translocation breakpoints recovered in B cells from KU70-/- mice (Boboila et al., 2010b). Shortly after, using an experimental system of chromosomal translocation induction based on zinc finger nuclease-induced DSBs in mouse cells, the group of Jasin reported that, while translocation breakpoints displayed less microhomologies in the absence of LIG3, LIG1 depletion did not affect microhomology use (Simsek et al., 2011). Note however that an in vitro study performed in human HTD114 cell line reported that both LIG1 and LIG3 were involved in MMEJ-dependent repair of an extrachromosomal DSB, although contribution of LIG3 appeared to be more important (Liang et al., 2008).

Altogether, data suggest the possible existence of two distinct alternative end-joining repair processes, both repressed by Ku70/Ku80 (Figure 2), The first one appears to rely on the presence of microhomologies for repair and I propose that it operates through a micro-SSA-type mechanism and involves LIG3. The second pathway of alternative end-joining does not appear to depend on pre-existing microhomologies and is believed to rely on LIG1. However, evidences for the conservation of the latter pathway throughout the eukaryotic lineage are still lacking.

\section{IMPLICATION OF ALTERNATIVE NHEJ IN TELOMERE FUSION EVENTS}

Telomeres at the end of linear chromosomes are reportedly resistant to end-to-end fusions thanks to the binding of socalled shelterin proteins (van Steensel et al., 1998). Accordingly, when normal shelterin density is breached, protection of telomeres against DNA damage activation is no longer ensured and DNA repair enzymes have access to telomeric DNA. Hence, loss of TRF2 shelterin component at mammalian telomeres induces telomere deprotection and LIG4-dependent fusions (Smogorzewska and de Lange, 2002). Further experiments revealed a direct role for mammalian RAP1/TRF2 complex in protection of telomeric DNA from NHEJ, independently of the involvement of TRF2 in telomeric-loop formation (Bae and Baumann, 2007).

Although NHEJ is clearly able to mediate end-to-end fusions in a telomere-deficient background, this DNA repair pathway is not required to catalyze all types of telomere fusions. Indeed, telomerase-deficient fission yeast mutants lacking either $\mathrm{Ku} 70$ or LIG4 display rearranged telomeres and chromosome circularization, indicating that alternative end-joining mechanisms are able to promote telomere fusion (Baumann and Cech, 2000). Similar conclusions were subsequently drawn from studies in budding yeast (Mieczkowski et al., 2003) and Arabidopsis (Riha and Shippen, 2003). A molecular analysis was then published in which authors analyzed telomere fusion events in Arabidopsis mutants lacking both TERT catalytic subunit of telomerase and Ku70 DNA repair protein (Heacock et al., 2004). Fusions between telomeric and subtelomeric regions of plant chromosomes were associated with large deletions, extending to more than $300 \mathrm{bp}$, and displayed overlapping homologies of up to 12 bp. Here too, Ku70 was acting as a strong inhibitor of the MMEJ-dependent mechanism of telomere fusion while MREll was found to promote fusions (Heacock et al., 2004). Subsequent work by the same group revealed that, as expected from an alternative end-joining mechanism, LIG4 was not required for plant telomere fusions (Heacock et al., 2007). Studies in human cells revealed similar mechanisms of telomere fusion in cells forced to divide in the absence of telomerase. In these cells, telomere fusions occurred with concomitant deletion of one or both telomeres and were characterized by the presence of microhomologies (Capper et al., 2007; Letsolo et al., 2010).

Following these in vitro studies, a report provided evidences for the involvement of both NHEJ and MMEJ repair pathways in mouse telomere fusion events in vivo (Rai et al., 2010). Using a combination of mutants and shRNA constructs, the authors showed that, while TRF2/RAP1 complex protects telomeres from ATM activation and NHEJ, single-stranded telomeric DNA-binding protein POT1, in conjunction with TPP1 shelterin component, inhibits ATR activation and alternative NHEJ. In agreement with previous data in human cells, their work further suggested that alternative NHEJ is the main pathway to process dysfunctional telomeres in mouse cells experiencing natural telomere erosion (Rai et al., 2010). Hence, despite a strong protection against NHEJ provided by TRF2, mammalian telomeres can be targets of MMEJ. Elegant demonstration of the role of shelterin components in telomere end protection was recently provided by the group of de Lange (Sfeir and de Lange, 2012). They confirmed the role of TRF2 as repressor of both ATM signaling and classical NHEJ and the role of POT1 in ATR signaling repression. They also showed that alternative NHEJ was repressed by various shelterin components as well as by Ku70/Ku80 and proposed that the redundancy of repressors may ensure better protection 


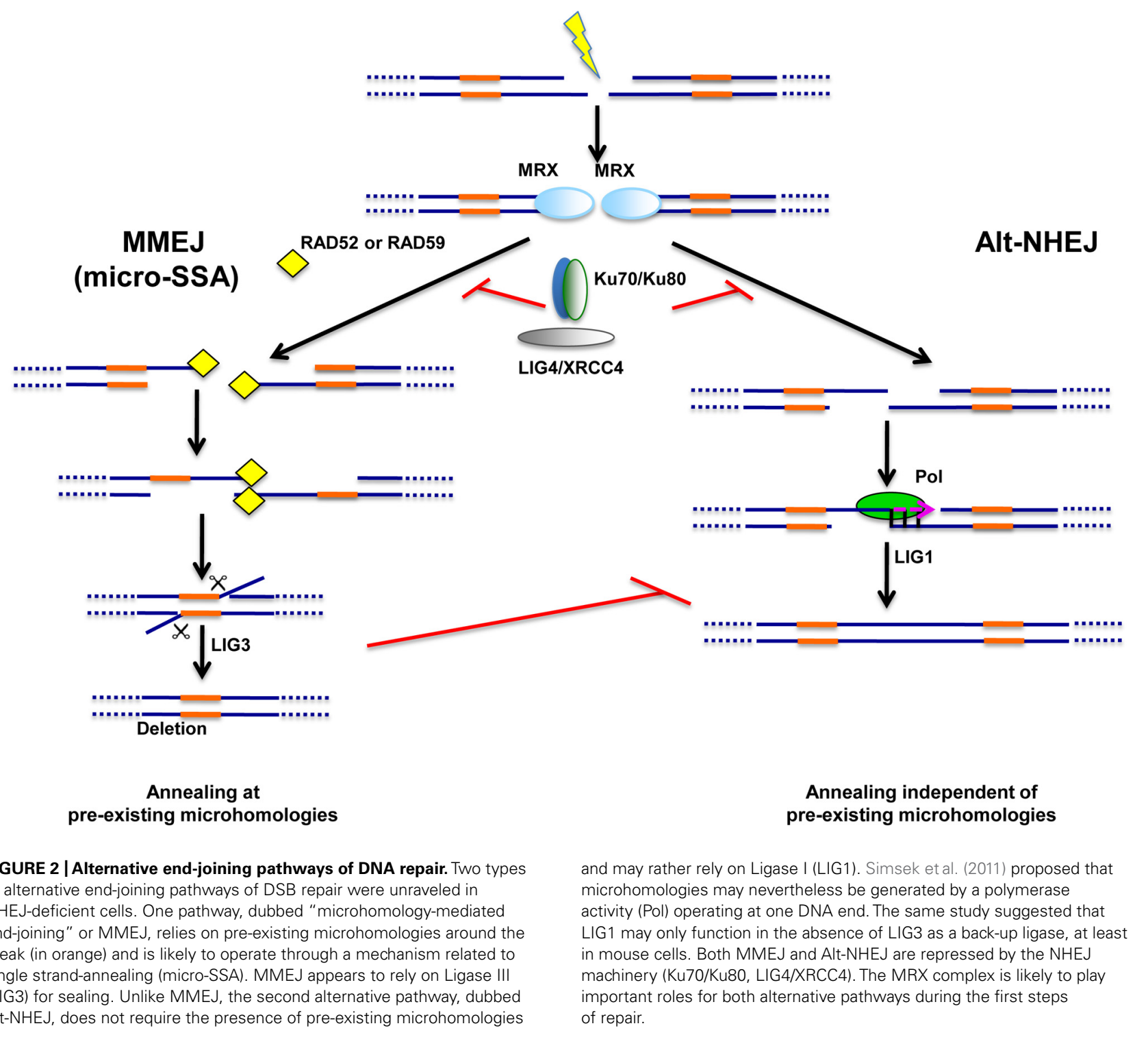

against dangerous alternative NHEJ at telomeres (Sfeir and de Lange, 2012).

Hence, the above studies clearly pointed toward an important contribution of alternative NHEJ to pathologic chromosome fusion events in cells with dysfunctional telomeres. Although evidences for an involvement of SSA proteins in these end-joining events is still lacking, telomere fusions are characterized by microhomologies at junctions, are repressed by $\mathrm{Ku} 70 / \mathrm{Ku} 80$ and rely on the MRE11 complex in plants and possibly also in human cells (Tankimanova et al., 2012).

\section{MUTAGENIC POTENTIAL OF ALTERNATIVE NHEJ IN MAMMALS}

In the late 1990s, it became evident that NHEJ acts as a tumor suppressing mechanism. Indeed, mice lacking both p53 and a NHEJ component, like DNA-PKcs, Ku80, XRCC4, or LIG4, were found to die in early postnatal life due to an elevated frequency of B cell

lymphomas displaying $I g H-M y c$ translocations and amplifications (reviewed in Sharpless et al., 2001). Importantly, these lymphomas were qualitatively distinct from those arising in a p53-deficient background alone as, in the latter mouse mutants, tumors had a later onset and did not generally harbor translocations. Following these observations, $I g H-M y c$ translocation junctions were recovered from $X R C C 4^{-/-} / p 53^{-/-}$mice in order to characterize the DNA repair mechanisms involved in chromosomal translocations. Sequencing of breakpoint junctions revealed the presence of microhomologous DNA sequences (Zhu et al., 2002; Wang et al., 2008). LIG4 haploinsufficiency was also reported to increase sarcoma formation in INK4a/ARF-/- mice by inducing chromosomal translocations, amplifications and deletions but translocation junctions were not characterized (Sharpless et al., 2001). In a more recent report, the group of F. Alt confirmed that an alternative end-joining pathway robustly catalyzes translocations in $\mathrm{KUTO}^{-/-} / \mathrm{LIG} 4^{-/-}$mice B cells that are fully deficient 
for classical NHEJ (Boboila et al., 2010b). However, as the authors did not detect any bias toward MMEJ at breakpoint junctions, they suggested that translocations were mediated by an alternative endjoining mechanism not relying on microhomologies. It should be tested whether this mutagenic alternative end-joining mechanism operating in B cells of $\mathrm{KUTO}^{-/-}$mice requires LIG1.

In human, an analysis of high-grade bladder carcinomas suggested that MMEJ may contribute to the high genomic instability of bladder cancer (Bentley et al., 2004). Indeed, authors showed that these tumors were highly proficient in their ability to perform MMEJ, even though Ku, DNA-PKcs and XRCC4 proteins were expressed at normal level.

\section{REFERENCES}

Audebert, M., Salles, B., and Calsou, P. (2004). Involvement of poly(ADP-ribose) polymerase-1 and XRCC1/DNA ligase III in an alternative route for DNA double-strand breaks rejoining. J. Biol. Chem. 279, 55117-55126.

Bae, N. S., and Baumann, P. (2007). A RAP1/TRF2 complex inhibits nonhomologous end-joining at human telomeric DNA ends. Mol. Cell 26, 323-334.

Baumann, P., and Cech, T. R. (2000). Protection of telomeres by the $\mathrm{Ku}$ protein in fission yeast. Mol. Biol. Cell 11, 3265-3275.

Bennardo, N., Cheng, A., Huang, N., and Stark, J. M. (2008). AltemativeNHEJ is a mechanistically distinct pathway of mammalian chromosome break repair. PLoS Genet. 4:e1000110. doi: 10.1371/journal. pgen. 1000110

Bentley, J., Diggle, C. P., Harnden, P., Knowles, M. A., and Kiltie, A. E. (2004). DNA double strand break repair in human bladder cancer is error prone and involves microhomology-associated end-joining. Nucleic Acids Res. 32, 5249-5259.

Boboila, C., Yan, C., Wesemann, D. R., Jankovic, M., Wang, J. H., Manis, J., et al. (2010a). Alternative end-joining catalyzes class switch recombination in the absence of both Ku70 and DNA ligase 4. J. Exp. Med. 207, 417-427.

Boboila, C., Jankovic, M., Yan, C. T., Wang, J. H., Wesemann, D. R., Zhang, T., et al. (2010b). Alternative endjoining catalyzes robust $\mathrm{IgH}$ locus deletions and translocations in the combined absence of ligase 4 and Ku70. Proc. Natl. Acad Sci. U.S.A. 107, 3034-3039.

Boboila, C., Oksenych, V., Gostissa, M., Wang, J. H., Zha, S., Zhang, Y., et al. (2012). Robust chromosomal DNA repair via alternative end-joining in the absence of X-ray repair crosscomplementing protein 1 (XRCC1).
Proc. Natl. Acad Sci. U.S.A. 109, 2473-2478.

Boulton, S., and Jackson, S. P. (1996). Saccharomyces cerevisiae Ku70 potentiates illegitimate DNA doublestrand break repair and serves as a barrier to error-prone DNA repair pathways. EMBO J. 15, 5083-5103.

Capper, R., Britt-Compton, B., Tankimanova, M., Rowson, J., Letsolo, B., Man, S., et al. (2007). The nature of telomere fusion and a definition of the critical telomere length in human cells. Genes Dev. 21, 2495-2508.

Chapman, J. R., Taylor, M. R. G., and Boulton, S. J. (2012). Playing the end game: DNA double-strand break repair pathway choice. Mol. Cell 47, 497-510.

Chapman, J. R., Barral, P., Vannier, J. B., Borel, V., Steger, M. Tomas-Loba, A., et al. (2013). RIF1 is essential for 53BP1-dependent nonhomologous end joining and suppression of DNA double-strand break resection. Mol. Cell 49, 858-871.

Decottignies, A. (2005). Capture of extranuclear DNA at fission yeast double-strand breaks. Genetics 171, 1535-1548.

Decottignies, A. (2007). Microhomology-mediated end joining in fission yeast is repressed by Pku70 and relies on genes involved in homologous recombination. Genetics 176, 14031415.

Delia-Maria, J., Zhou, Y., Tsai, M.-S., Kuhnlein, J., Carney, J. P., Paull, T. T., et al. (2011). Human Mre11/Human Rad50/Nbs1 and DNA Ligase IIIa/XRCCl protein complexes act together in an alternative nonhomologous end joining pathway. J. Biol. Chem. 286, 3384533853.

Di Virgilio, M., Callen, E., Yamane, A., Zhang, W., Jankovic, M., Gitlin, A. D., et al. (2013). Rif1 prevents resection of DNA breaks and promotes

Altogether, data reported so far indicate that, although the classical LIG4/Ku-dependent NHEJ pathway appears to act as a potent tumor suppressor mechanism, alternative end-joining pathways, whether relying on microhomologies or not, promote chromosomal translocations. In the future, it would be interesting to better characterize these alternative pathways of end-joining and to identify the genes involved in the repair processes.

\section{ACKNOWLEDGMENTS}

This work was supported by the Fonds National de la Recherche Scientifique (FNRS), Belgium. I am grateful to Patrick Revy (Hôpital Necker, Paris, France) for critical reading of the manuscript.

immunoglobulin class switching. Science 339, 711-715.

Escribano-Diaz, C., Orthwein, A. Fradet-Turcotte, A., Xing, M., Young, J. T., Tkác, J., etal. (2013). A cell cycle-dependent regulatory circuit composed of 53BP1-RIF1 and BRCA1-CtIP controls DNA repair pathway choice. Mol. Cell 49, 872-883.

Feldmann, E., Schmiemann, V., Goedecke, W., Reichenberger, S., and Pfeiffer, P. (2000). DNA doublestrand break repair in cell-free extracts from Ku80-deficient cells: implications for $\mathrm{Ku}$ serving as an alignment factor in non-homologous DNA end joining. Nucleic Acids Res. 32, 2585-2596.

Gottlich, B., Reichenberger, S., Feldmann, E., and Pfeiffer, P. (1998). Rejoining of DNA double-strand breaks in vitro by single-strand annealing. Eur. J. Biochem. 258, 387395.

Heacock, M., Idol, R. A., Friesner, J. D., Britt, A. B., and Shippen, D. E. (2007). Telomere dynamics and fusion of critically shortened telomeres in plants lacking DNA ligase IV. Nucleic Acids Res. 35, 6490-6500.

Heacock, M., Spangler, E., Riha, K., Puizina, J., and Shippen, D. E. (2004). Molecular analysis of telomere fusions in Arabidopsis: multiple pathways for chromosome end-joining. EMBO J. 23 2304-2313.

Kabotyanski, E. B., Gomelsky, L. Han, J.-O., Stamato, T. D., and Roth, D. B. (1998). Double-strand break repair in Ku86- and XRCC4deficient cells. Nucleic Acids Res. 26, 5333-5342.

Kramer, K. M., Brock, J. A., Bloom, K., Moore, J. K., and Haber, J. E. (1994). Two different types of double-strand breaks in Saccharomyces cerevisiae are repaired by similar RAD52-independent, nonhomologous recombination events. Mol. Cell. Biol. 14, 1293-1301.
Letsolo, B. T., Rowson, J., and Baird, D. M. (2010). Fusion of short telomeres in human cells is characterized by extensive deletion and microhomology, and can result in complex rearrangements. Nucleic Acids Res. $38,1841-1852$.

Liang, L., Deng, L., Nguyen, S. C., Zhao, X., Maulion, C. D., Shao, C., et al. (2008). Human DNA ligases I and III, but not ligase IV, are required for microhomology-mediated end joining of DNA double-strand breaks. Nucleic Acids Res. 36, 3297-3310.

Ma, J.-L., Kim, E. M., Haber, J. E., and Lee, S. E. (2003). Yeast Mre 11 and Rad 1 proteins define $\mathrm{Ku}$ independent mechanism to repair double-strand breaks lacking overlapping end sequences. Mol. Cell. Biol. 23, 8820-8828.

Mason, R. M., Thacker, J., and Fairman, M. P. (1996). The joining of non-complementary DNA double-strand breaks by mammalian extracts. Nucleic Acids Res. 24, 49464953.

Mieczkowski, P. A., Mieczkowska, J. O., Dominaska, M., and Petes, T. D. (2003). Genetic regulation of telomere-telomere fusions in the yeast Saccharomyces cerevisiae. Proc. Natl. Acad. Sci. U.S.A. 100, 1085410859.

Paques, F., and Haber, J. E. (1999). Multiple pathways of recombination induced by double-strand breaks in Saccharomyces cerevisiae. Microbiol. Mol. Biol. Rev. 63, 349-404.

Pierce, A. J., Hu, P., Han, M., Ellis, N., and Jasin, M. (2001). Ku DNA endbinding protein modulates homologous repair of double-strand breaks in mammalian cells. Genes Dev. 15, 3237-3242.

Rai, R., Zheng, H., He, H., Luo, Y., Multani, A., Carpenter, P. B., et al. (2010). The function of classical and alternative non-homologous end-joining pathways in the fusion of dysfunctional telomeres. EMBO J. 29, 2598-2610. 
Riha, K., and Shippen, D. E. (2003). $\mathrm{Ku}$ is required for telomeric C-rich strand maintenance, but not for endto-end chromosome fusions in Arabidopsis. Proc. Natl. Acad. Sci. U.S.A. 100, 611-615.

Rijkers, T., Van Den Ouwenland, J., Morolli, B., Rolink, A. G., Baarends, W. M., Van Sloun, P. P. H., etal. (1998). Targeted inactivation of mouse RAD52 reduces homologous recombination but not resistance to ionizing radiation. Mol. Cell. Biol. 18 , 6423-6429.

Roth, D. B., Porter, T. N., and Wilson, J. H. (1985). Mechanisms of nonhomologous recombination in mammalian cells. Mol. Cell. Biol. 5, 2599-2607.

Roth, D. B., and Wilson, J. H. (1986). Nonhomologous recombination in mammalian cells: role for short sequence homologies in the joining reaction. Mol. Cell. Biol. 6, 42954304.

Sfeir, A., and de Lange, T. (2012). Removal of shelterin reveals the telomere end-protection problem. Science 336, 593-597.

Sharpless, N. E., Ferguson, D. O., O’Hagan, R. C., Castrillon, D. H., Lee, C., Farazi, P. A., et al. (2001). Impaired nonhomologous end-joining provokes soft tissue sarcomas harboring chromosomal translocations, amplifications, and deletions. Mol. Cell 8, 1187-1196.

Simsek, D., Brunet, E., Wong, S. YW., Katyal, S., Gao, Y., McKinnon, P. J., etal. (2011). DNA Ligase
III promotes alternative nonhomologous end-joining during chromosomal translocation formation. PLoS Genet. 7:el002080. doi: 10.1371/journal.pgen.1002080

Smogorzewska, A., and de Lange, T. (2002). Different telomere damage signaling pathways in human and mouse cells. EMBO J. 21, 4338-4348.

Soulas-Sprauel, P., Le Guyader, G. Rivera-Munoz, P., Abramowski, V., Olivier-Martin, C., Goujet-Zalc, C. et al. (2007). Role for DNA repair factor XRCC4 in immunoglobulin class switch recombination. J. Exp. Med. 204, 1717-1727.

Stark, J. M., Pierce, A. J., Oh, J. Pastink, A., and Jasin, M. (2004). Genetic steps of mammalian homologous repair with distinct mutagenic consequences. Mol. Cell. Biol. 24 9305-9316.

Sugawara, N., Ira, G., and Haber, J. E. (2000). DNA length dependence of the single-strand annealing pathway and the role of Saccharomyces cerevisiae RAD59 in double-strand break repair. Mol. Cell. Biol. 20, 53005309.

Symington, L. S., and Gautier, J. (2011) Double-strand break resection and repair pathway choice. Annu. Rev. Genet. 45, 247-271.

Tankimanova, M., Capper, R., Letsolo, B. T., Rowson, J., Jones, R. E., Britt-Compton, B., et al. (2012). Mre11 modulates the fidelity of fusion between short telomeres in human cells. Nucleic Acids Res. 40, 2518-2526. van Steensel, B., Smogorzewska, A., and de Lange, T. (1998). TRF2 protects human telomeres from end-to-end fusions. Cell 92, 401-413.

Wang, H., Perrault, A. R., Takeda, Y., Qin, W., Wang, H., and Iliakis, G. (2003). Biochemical evidence for Ku-independent backup pathways of NHEJ. Nucleic Acids Res. 31, 5377 5388.

Wang, H., Rosidi, B., Perrault, R., Wang, M., Zhang, L., Windhofer, F., et al (2005). DNA Ligase III as a candidate component of backup pathways of nonhomologous end joining. Cancer Res. 65, 4020-4030.

Wang, J. H., Alt, F. W., Gostissa, M. Datta, A., Murphy, M., Alimzhanov, M. B., et al. (2008). Oncogenic transformation in the absence of Xrcc4 targets peripheral B cells that have undergone editing and switching. $J$. Exp. Med. 205, 3079-3080.

Weterings, E., and Chen, D. J. (2008). The endless tale of non-homologous end-joining. Cell Res. 18, 114-124.

Yan, C. T., Boboila, C., Souza, E. K., Franco, S., Hickernell, T. R., Murphy, M., et al. (2007). IgH class switching and translocations use a robust nonclassical end-joining pathway. Nature $449,478-482$

Yu, X., and Gabriel, A. (2003). Kudependent and Ku-independent end-joining pathways lead to chromosomal rearrangements during double-strand break repair in Saccharomyces cerevisiae. Genetics 163 843-856.
Zhu, C., Mills, K. D., Ferguson, D O., Lee, C., Manis, J., Fleming, J., etal. (2002). Unrepaired DNA breaks in p53-deficient cells lead to oncogenic gene amplification subsequent to translocations. Cell 109, 811-821.

Zimmermann, M., Lottersberger, F., Buonomo, S. B., Sfeir, A., and de Lange, T. (2013). 53BP1 regulates DSB repair using Rif1 to control $5^{\prime}$ end resection. Science 339, 700-704.

Conflict of Interest Statement: The author declares that the research was conducted in the absence of any commercial or financial relationships that could be construed as a potential conflict of interest.

Received: 20 February 2013; paper pending published: 12 March 2013; accepted: 15 March 2013; published online: 02 April 2013.

Citation: Decottignies A (2013) Alternative end-joining mechanisms: a historical perspective. Front. Genet. 4:48. doi: 10.3389/fgene.2013.00048

This article was submitted to Frontiers in Cancer Genetics, a specialty of Frontiers in Genetics.

Copyright (c) 2013 Decottignies. This is an open-access article distributed under the terms of the Creative Commons Attribution License, which permits use, distribution and reproduction in other forums, provided the original authors and source are credited and subject to any copyright notices concerning any thirdparty graphics etc. 